\title{
Long Non-Coding RNA CRNDE Regulates Angiogenesis in Hepatoblastoma by Targeting the MiR-203/VEGFA Axis
}

\author{
Li-Jian Chen Miao-Xian Yuan Chun-Yi Ji Yan-Bing Zhang Yu-Ming Peng \\ Tian Zhang Hong-Qiang Gao Xin-Yi Sheng Zhao-Yang Liu Wei-Xin Xie \\ Qiang Yin
}

Department of General Surgery, Hunan Children's Hospital, Changsha, PR China

\section{Keywords}

Angiogenesis · Hepatoblastoma · CRNDE · miR-203 · VEGFA

\begin{abstract}
Objective: MiR-203 has been shown to participate in multiple malignancies, but the role of miR-203 in hepatoblastoma $(\mathrm{HB})$ remains unclear. The aim of our study was to investigate the effects of miR-203 in HB. Methods: A total of 15 pairs of $\mathrm{HB}$ tissues and para-tumour normal tissues were collected for the experiments. RT-qPCR and Western blotting were performed to detect the expression of CRNDE, miR-203, and VEGFA at the mRNA and/or protein levels, respectively. A dual luciferase assay verified the target relationship between miR-203 and the 3'UTR of VEGFA as well as miR-203 and CRNDE. In addition, MTT, wound healing, and tube formation assays were performed to assess the effects of miR-203, VEGFA, and CRNDE on cell proliferation, migration, and angiogenesis, respectively. Results: Our data revealed that miR-203 expression was decreased in HB tissues, while long non-coding RNA (IncRNA) CRNDE expression was increased. The dysregulation of miR-203 and CRNDE was closely related to tumour size and stage. Moreover, overexpression of miR-
\end{abstract}

karger@karger.com

www.karger.com/pat

Karger
203 inhibited angiogenesis. A dual luciferase assay verified that VEGFA is a direct target of miR-203 and that CRNDE binds to miR-203. Furthermore, our results showed that miR203 suppressed cell viability, migration, and angiogenesis by regulating VEGFA expression. Additionally, it was confirmed that CRNDE promoted angiogenesis by negatively regulating miR-203 expression. Conclusion: IncRNA CRNDE targets the miR-203/VEGFA axis and promotes angiogenesis in HB. These results provide insight into the underlying mechanisms of $\mathrm{HB}$ and indicate that CRNDE and miR-203 might be potential targets for HB therapy.

๑) 2020 S. Karger AG, Basel

\section{Introduction}

Hepatoblastoma ( $\mathrm{HB})$ is the most common primary liver malignant tumour in childhood [1]. In contrast to hepatocellular carcinoma, $\mathrm{HB}$ pathogenesis is not correlated with viral hepatitis, liver cirrhosis, or other underlying liver lesions [2]. The abnormal expression of genes is closely related to the process of tumour formation and progression [3]. The potential role of long non-coding 
RNAs (lncRNAs) and microRNAs (miRNAs) in the development of $\mathrm{HB}$ has been gradually revealed $[4,5]$, but the underlying molecular mechanisms have not been fully understood. Therefore, the identification of novel targets and abnormal signalling pathways in HB is urgently needed for the development of effective cancer diagnosis and treatment.

Rapidly developing transcriptomics analysis technology in recent years has revealed the potential regulatory mechanisms of lncRNAs in various diseases, especially in tumours [6,7]. lncRNAs can affect the proliferation and metastasis of cells by regulating gene promoter activity by sponging specific miRNAs, which are important factors in tumourigenesis $[8,9]$. Accumulating evidence supports that lncRNAs and miRNAs make up a regulatory network. For instance, lncRNAs could combine with miRNAs as a target-mimetic sponge, thereby inhibiting the bio-functions of miRNAs [10]. Therefore, dysregulation of lncRNAs participates in the occurrence and development of cancers. For example, lncRNA SNHG10 was reported to facilitate hepatocarcinogenesis and metastasis [11]. Another study revealed that knockdown of LUCAT1 inhibited cell proliferation, migration, and invasion [12]. A previous study reported that lncRNA CRNDE was overexpressed in HB compared to normal liver tissue, suggesting that lncRNA CRNDE plays a potential role in HB pathogenesis [13]. However, the biological function and clinical significance of CRNDE in $\mathrm{HB}$ remains unclear.

miRNAs are small non-protein-coding sequences that consist of approximately 20-24 nucleotides and suppress target mRNA translation by post-transcriptionally binding to the $3^{\prime}$ untranslated region ( $\left.3^{\prime} \mathrm{UTR}\right)$. Previous reports have suggested that miRNAs are involved in the regulation of a variety of physiological and pathological processes, including tumour growth, migration, and angiogenesis [14-16]. MiR-203 has been demonstrated to be downregulated in a number of malignant diseases, including bladder cancer, cervical cancer, breast cancer, prostate cancer, and hepatocellular carcinoma [17-21]. Furthermore, Zhu et al. [22] reported that miR-203 could act as a tumour suppressor by inhibiting tumour cell proliferation and angiogenesis in cervical cancer, which suggested that miR-203 might be a candidate therapeutic target and prognostic biomarker. However, whether miR203 could regulate angiogenesis in HB remains unclear.

The aim of our research was to investigate the expression and function of CRNDE and miR-203 in HB. In this study, we found that miR-203 was hypoexpressed in $\mathrm{HB}$ tissues compared to adjacent normal tissues, while
CRNDE expression was increased in HB tissues. We further demonstrated that CRNDE regulated angiogenesis by targeting the miR-203/VEGFA axis. Our results indicated that the CRNDE/miR-203/VEGFA axis could be a potential therapeutic target in $\mathrm{HB}$.

\section{Materials and Methods}

\section{Patients and Samples}

A total of 15 pairs of $\mathrm{HB}$ tissues and para-tumour normal tissues were collected from July 2010 to July 2018 at Hunan Children's Hospital. The specimens were obtained from each patient or direct relatives.

\section{Cell Culture}

The HB cell line HuH-6 was obtained from the Chinese Academy of Science Cell Bank (\#TCHu181, Shanghai, China) and maintained in DMEM (Invitrogen, Carlsbad, CA, USA) with 10\% FBS (Invitrogen). Human umbilical vein endothelial cells (HUVECs) were purchased from the American Type Culture Collection (\#1730, ATCC, USA) and maintained in DMEM (Invitrogen) with $10 \%$ FBS (Invitrogen). In this study, HUVECs were treated with different groups of HuH-6 cell supernatants.

\section{Cell Transfection}

Si-CRNDE, miR-203 mimics, negative control (NC) mimics, miR-203 inhibitor, NC inhibitor, pmirGLO-VEGFA 3'UTR WT, pmirGLO-VEGFA 3'UTR MUT, pcDNA3.1-VEGFA (p-VEGFA), and pcDNA3.1-NC were provided by RiboBio (Guangzhou, China). According to the manufacturer's instructions, plasmids or oligonucleotides were transfected into cells along with Lipofectamine $^{\mathrm{TM}} 2000$ (Invitrogen).

\section{Total RNA Extraction and Quantitative Real-Time PCR} Analysis

Total RNA was extracted using TRIzol reagent (Invitrogen) following the manufacturer's recommendations. cDNA was synthesized using the RT Reagent Kit (Transgen, Beijing, China) according to the manufacturer's instructions. For the quantification of gene expression, quantitative real-time PCR (qRT-PCR) was performed using an ABI Detection System (Applied Biosystems, Inc., USA) with Reverse Transcription Kit (TaKaRa, Japan). The primers used for qRT-PCR are as follows:

CRNDE-F 5'-CGATCGCGCTATTGTCATGG-3'

CRNDE-R 5' -TCCGCCTCGCTTAGACATTG-3';

miR-203-F $5^{\prime}$-GGGGTGAAATGTTTAGGAC-3'

miR-203-R 5'-CAGTGCGTGTCGTGGAGT-3';

VEGFA-R 5'-CACCAAGGCCAGCACATAGG-3'

VEGFA-F $5^{\prime}$-AGGGAGGCTCCAGGGCATTA-3';

GAPDH-F 5'-AGGTCGGTGTGAACGGATTTG-3'

GAPDH-R 5'-GGGGTCGTTGATGGCAACA-3';

U6-F 5' -CGCTTCGGCAGCACATATACTA-3'

U6-R 5'-CGCTTCACGAATTTGCGTGTCA-3'.

Total Protein Extraction and Western Blotting

After treatment with miR-203 inhibitor, miR-203 mimics, siCRNDE, or si-NC, HuH-6 cells were washed with cold PBS and lysed with RIPA lysis buffer (Keygen, China). Then, total protein 
Fig. 1. miR-203 expression is decreased in $\mathrm{HB}$ tissues, and overexpression of miR203 suppressed angiogenesis. a qRT-PCR showed that miR-203 is downregulated in HB tissues compared with para-tumour normal tissues. $\mathbf{b}$ The expression of miR203 decreased in groups III and IV compared with groups I and II. In addition, the expression of miR-203 decreased when the tumour size was greater than 5 . The expression of miR-203 was not related to age or gender. c The expression of miR-203 was significantly increased in $\mathrm{HuH}-6$ cells treated with miR-203 mimics. d The MTT assay suggested that overexpression of miR-203 suppressed the proliferation of HUVECs. e The wound healing assay showed that overexpression of miR-203 suppressed the migration of HUVECs. $\mathbf{f}$ The tube formation assay suggested that overexpression of miR-203 suppressed the angiogenesis of HUVECs.
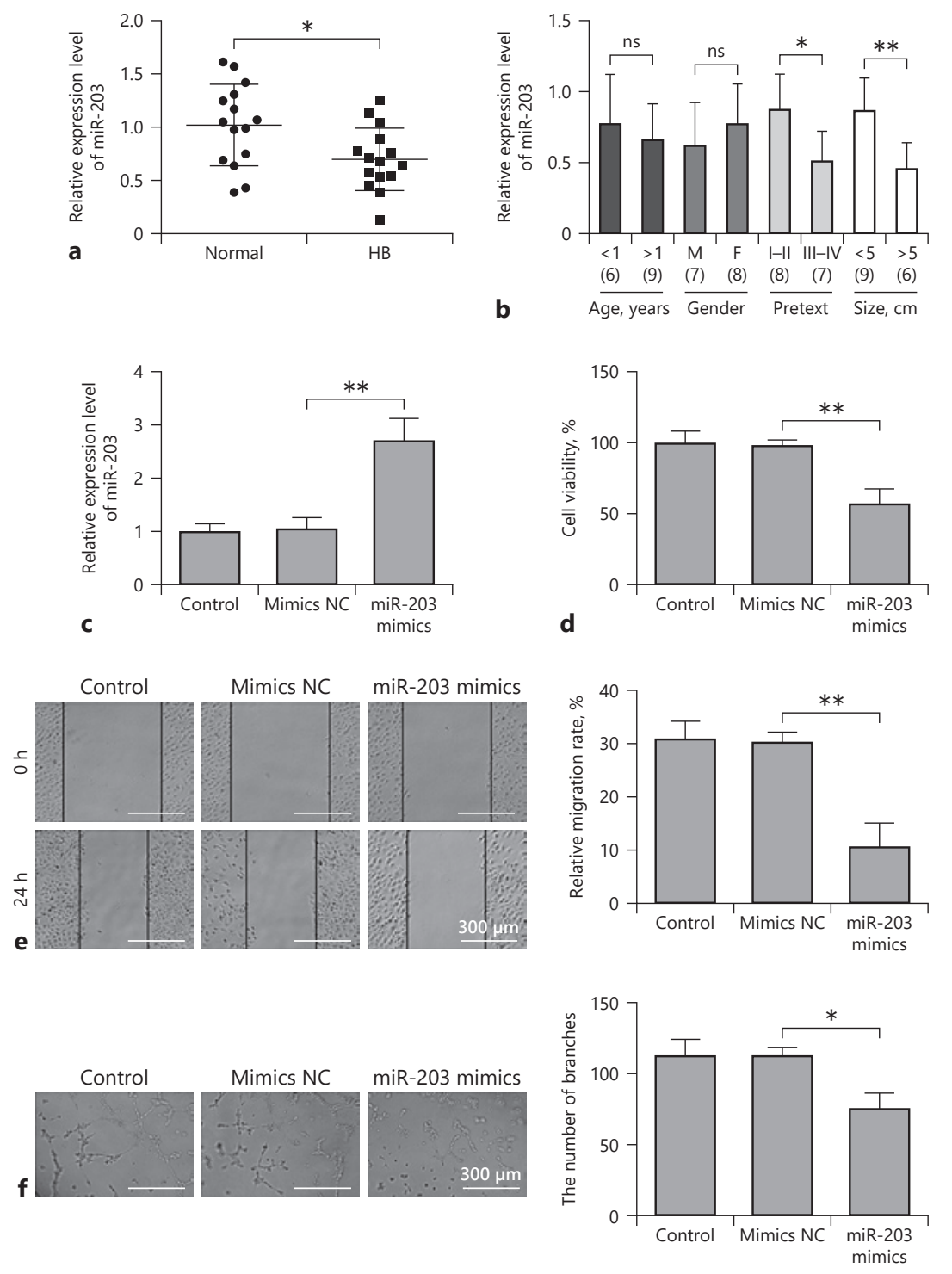

was isolated with a total protein isolation kit (Keygen), followed by quantification using a BCA kit (Keygen). After denaturation, samples were separated using 10\% SDS-PAGE and electro-transferred onto a PVDF membrane using the Protean Electrotransfer System (Bio-Rad, USA). Then, PVDF membranes were incubated with $5 \%$ skim milk in PBST for $1 \mathrm{~h}$ and incubated overnight at $4{ }^{\circ} \mathrm{C}$ with the specific primary antibody anti-VEGFA (rabbit monoclonal, \#ab52917, Abcam, USA, 1:2,000) or anti-GAPDH (rabbit monoclonal, \#ab181602, Abcam, 1:3,000). The membrane was washed with TBST solution 3 times. Next, PVDF membranes were blotted with an appropriate peroxidase-conjugated secondary antibody (goat anti-rabbit polyclonal secondary antibody, \#ab150077, Abcam, $1: 2,000$ ) incubated at $37^{\circ} \mathrm{C}$ for $1 \mathrm{~h}$. After the final PBS washing, the target proteins were detected and quantified via enhanced chemiluminescence (Advansta, USA) and analysed with ImageJ software.

\section{MTT Assay for Cell Proliferation}

HUVECs were harvested and seeded into 96-well plates and then cultured with medium from $\mathrm{HuH}-6$ cells for $24 \mathrm{~h}$. Then, HUVECs were treated with fresh medium containing $5 \mathrm{mg} / \mathrm{mL}$ MTT (Sigma, USA) for $3 \mathrm{~h}$, followed by treatment with $200 \mu \mathrm{L}$ DMSO. Finally, the absorbance of each plate was detected by a microplate reader at $490 \mathrm{~nm}$.

\section{Wound Healing Assay}

HUVECs were seeded and cultured in 6-well plates, and then treated with conditioned medium from HuH- 6 cells for $24 \mathrm{~h}$. Then, scratches were created on the cell monolayer using a $10-\mu \mathrm{L}$ pipette tip. Images of the scratches were obtained using a microscope (Nikon, Japan) at 0 and $24 \mathrm{~h}$ after scratching. Finally, migration distances were measured and analysed using Image-Pro Plus software (Media Cybernetics, Inc., USA). 


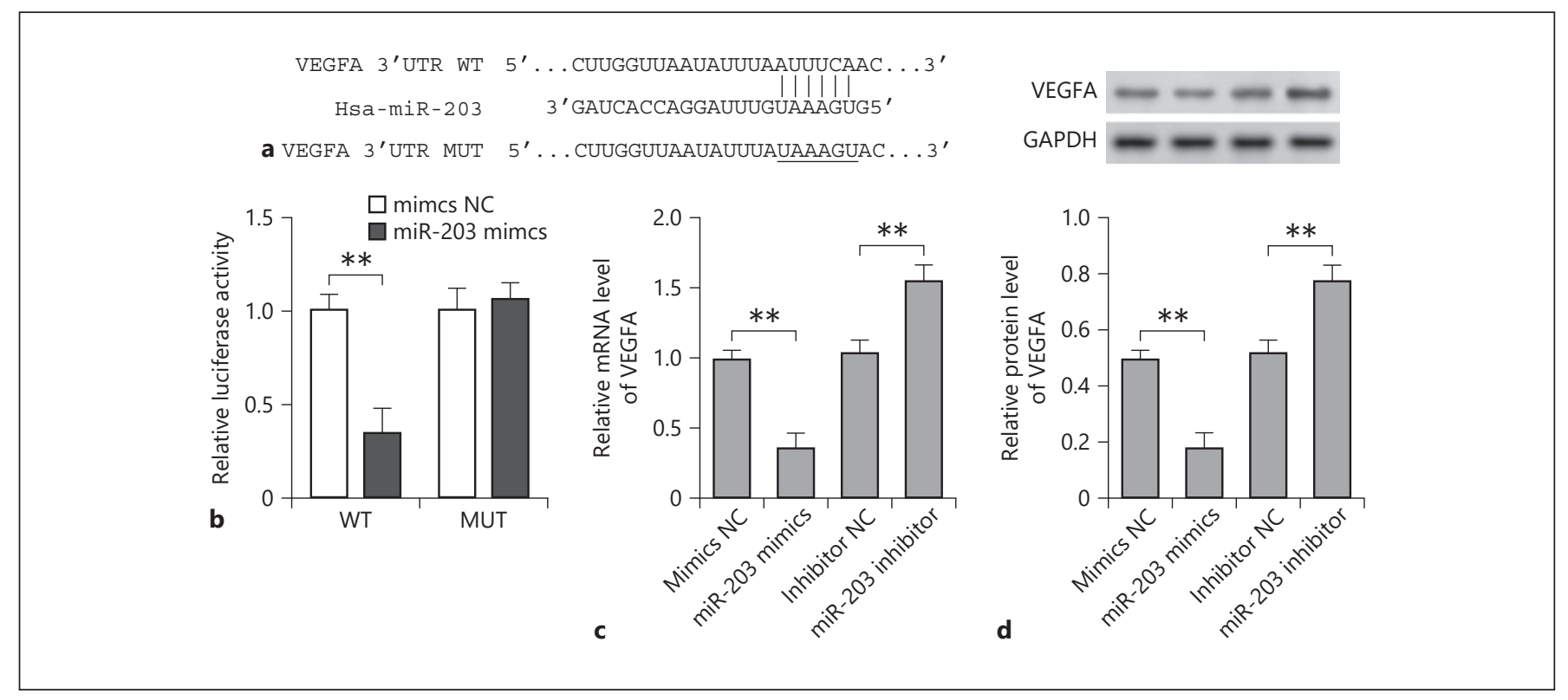

Fig. 2. MiR-203 targeted and regulated VEGFA expression. a Bioinformatic analysis demonstrated the targeting association between the $3^{\prime}$ UTR of VEGFA mRNA and miR-203. b Transfection of miR-203 mimics significantly reduced the luciferase activity of the wild-type VEGFA reporter (WT) but not the mutant reporter (MUT). c qRT-PCR results showed that VEGFA expression was

Tube Formation Assay

HuH-6 cells were treated with miR-203 mimics, miR-203 mimics NC, miR-203 inhibitor, si-NC, si-CRNDE, p-NC, p-VEGFA, or $\mathrm{NC}$ for $24 \mathrm{~h}$ and then cultured with medium without FBS for $24 \mathrm{~h}$. Then, the above medium was collected. HUVECs were resuspended in the above medium and seeded into 96-well plates pre-coated with Matrigel (BD Biosciences). After incubation, the medium was removed and replaced by $50 \mu \mathrm{L}$ calcein-AM. Finally, tube formation was observed using a phase contrast microscope (Olympus, Japan).

\section{Dual Luciferase Reporter Assay}

A dual luciferase reporter assay was performed to determine the interaction between miR-203 and the $3^{\prime}$ UTR of VEGFA or CRNDE. The vector pmirGLO was obtained from the Promega company (Promega, USA). The plasmids were synthesized as pmirGLO-VEGFA or CRNDE-WT and pmirGLO-VEGFA or CRNDE-MUT. The HB cell line HuH-6 was seeded on 24-well plates and transfected with $300 \mathrm{ng} /$ well of luciferase vectors encoding the wild-type (WT) or mutant (MUT) 3'UTR of VEGFA or CRNDE together with miR-203 mimics or NC mimics (Applied Biosystems) using Lipofectamine 2000 reagent (Invitrogen). Twenty-four hours after co-transfection, the luciferase activities were detected with a Dual-Luciferase Reporter Assay System (Promega).

\section{Statistical Analysis}

The data are expressed as the mean \pm standard deviation. Statistical significance among multiple groups was analysed with one- significantly decreased in HuH-6 cells treated with miR-203 mimics but increased in the miR-203 inhibitor group. d Western blotting showed that VEGFA expression was significantly decreased in HuH-6 cells treated with miR-203 mimics but increased in the miR-203 inhibitor group.

way ANOVA followed by the Newman-Keuls post hoc test. Comparisons between two groups were analysed using Student's $t$ test. $p<0.05$ was considered statistically significant. All results were obtained from at least three independent experiments.

\section{Results}

\section{MiR-203 Is Decreased in HB Tissues and Suppresses} the Viability, Migration, and Angiogenesis of

\section{HUVECs}

To explore whether miR-203 was abnormally expressed in HB tissues, we performed qRT-PCR to detect the miR-203 expression level in $15 \mathrm{HB}$ tissues and 15 adjacent normal tissues. As shown in Figure 1a, miR-203 was significantly downregulated in $\mathrm{HB}$ tissues compared to adjacent normal tissues. According to a comparison between PRETEXT stage and miR-203 expression, the expression of miR-203 decreased in groups III and IV compared to groups I and II. In addition, the expression of miR-203 decreased when the tumour size was greater than $5 \mathrm{~cm}$. The expression of miR-203 was not related to age or gender (Fig. 1b). To detect the potential role of miR-203 in angiogenesis, $\mathrm{HuH}-6$ cells 


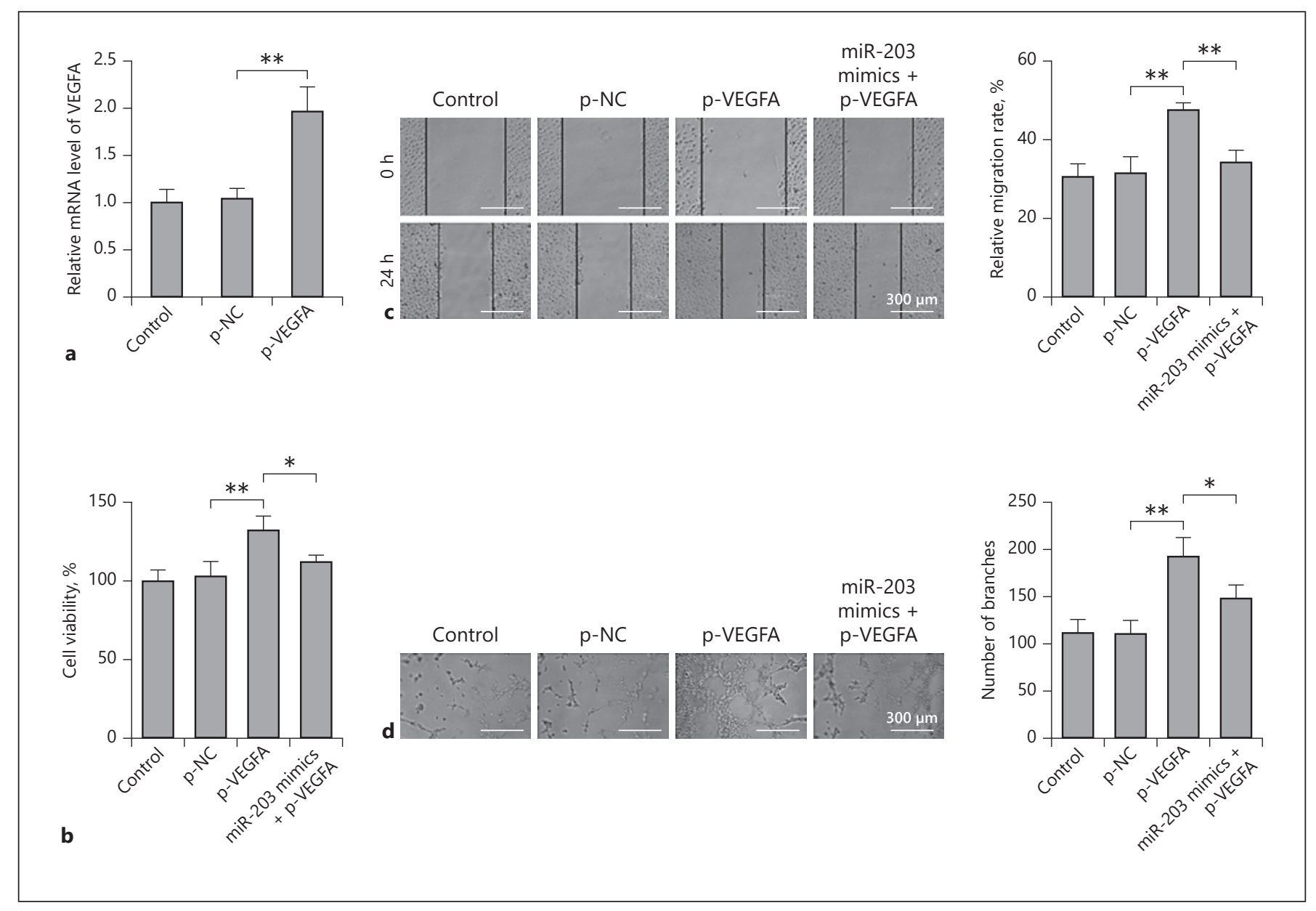

Fig. 3. MiR-203 suppressed angiogenesis by regulating VEGFA expression. a The expression of VEGFA was significantly increased in HuH-6 cells transfected with p-VEGFA. b The MTT assay suggested that overexpression of VEGFA promoted the proliferation of HUVECs, but the effect was reversed by overexpression of miR203. c The wound healing assay suggested that overexpression of
VEGFA promoted the migration of HUVECs, but the effect was reversed by overexpression of $\mathrm{miR}-203$. $\mathbf{d}$ The tube formation assay suggested that overexpression of VEGFA promoted the angiogenesis of HUVECs, but the effect was reversed by overexpression of miR-203. were transfected with NC mimics or miR-203 mimics. As shown in Figure 1c, the qRT-PCR results demonstrated that miR-203 mimics significantly increased the expression of miR-203 in HuH-6 cells. Furthermore, MTT, wound healing, and tube formation assays were performed to detect the viability, in vitro migration capacity and angiogenesis of HUVECs. The viability (Fig. 1d) and migration (Fig. 1e) of HUVECs were dramatically decreased after treatment with medium from HuH-6 cells transfected with miR-203 mimics. In addition, the tube formation assay suggested that overexpression of miR-203 reduced angiogenesis (Fig. 1f). These results indicate that miR-203 suppresses the angiogenesis of HUVECs.

miR-203 Regulates Angiogenesis in $\mathrm{HB}$
VEGFA Is a Direct Target of miR-203

To further investigate the specific molecular mechanism of how miR-203 regulates angiogenesis in $\mathrm{HuH}-6$ cells, potential target genes were predicted by bioinformatics methods, especially focusing on genes involved in angiogenesis. As shown in Figure 2a, a binding site of miR203 was found in the $3^{\prime}$ UTR of VEGFA. To confirm that VEGFA is a direct target of miR-203, HuH-6 cells were co-transfected with miR-203 mimic or mimic NC and MUT-VEGFA (pmirGLO-VEGFA 3'UTR MUT) or WTVEGFA (pmirGLO-VEGFA 3'UTR WT). Then, the dual luciferase reporter assay was carried out in $\mathrm{HuH}-6$ cells. According to Figure $2 \mathrm{~b}$, miR-203 decreased the luciferase activity of WT-VEGFA but not MUT-VEGFA. Further- 
more, to confirm that VEGFA was negatively regulated by miR-203, HuH-6 cells were transfected with miR-203 mimics or miR-203 inhibitors. Subsequently, qRT-PCR analysis and Western blotting assays were applied to assess the mRNA and protein levels of VEGFA expression, respectively. The results indicated that overexpression of miR-203 significantly inhibited the expression of VEGFA, while downregulation of miR-203 significantly promoted the expression of VEGFA (Fig. 2c, d). The above data suggested that miR-203 could directly bind to the VEGFA $3^{\prime}$ UTR and negatively regulate VEGFA expression.

\section{MiR-203 Inhibits Angiogenesis by Regulating VEGFA} Expression

Subsequently, to investigate whether miR-203 regulated angiogenesis through regulating the expression of
VEGFA, HuH-6 cells were transfected with p-NC (pVEGFA NC), p-VEGFA, or p-VEGFA + miR-203 mimics. The expression of VEGFA was dramatically increased after treatment with p-VEGFA compared with the p-NC group (Fig. 3a). In addition, the medium from different groups (controls, p-NC, p-VEGFA, and p-VEGFA + miR-203 mimics) was collected and used to culture HUVECs for $24 \mathrm{~h}$. According to Figure 3b, the MTT assay showed that high levels of VEGFA promoted the viability of HUVECs, which was significantly decreased in the pVEGFA + miR-203 mimic group. A wound healing assay suggested that overexpression of VEGFA could promote the migration of HUVECs, which was reversed by cotransfection of miR-203 mimic and p-VEGFA (Fig. 3c). The tube formation assay showed that overexpression of VEGFA in HuH-6 cells could promote angiogenesis,
Fig. 4. CRNDE targeted and negatively regulated the expression of miR-203. a Bioinformatic analysis demonstrated the targeting association between the 3'UTR of CRNDE and miR-203. b Transfection of miR-203 mimics significantly reduced the luciferase activity of the wild-type CRNDE but not the mutant. c The expression of CRNDE was significantly decreased after transfection with si-CRNDE in $\mathrm{HuH}-6$ cells. d miR-203 expression was significantly increased in $\mathrm{HuH}-6$ cells transfected with si-CRNDE.

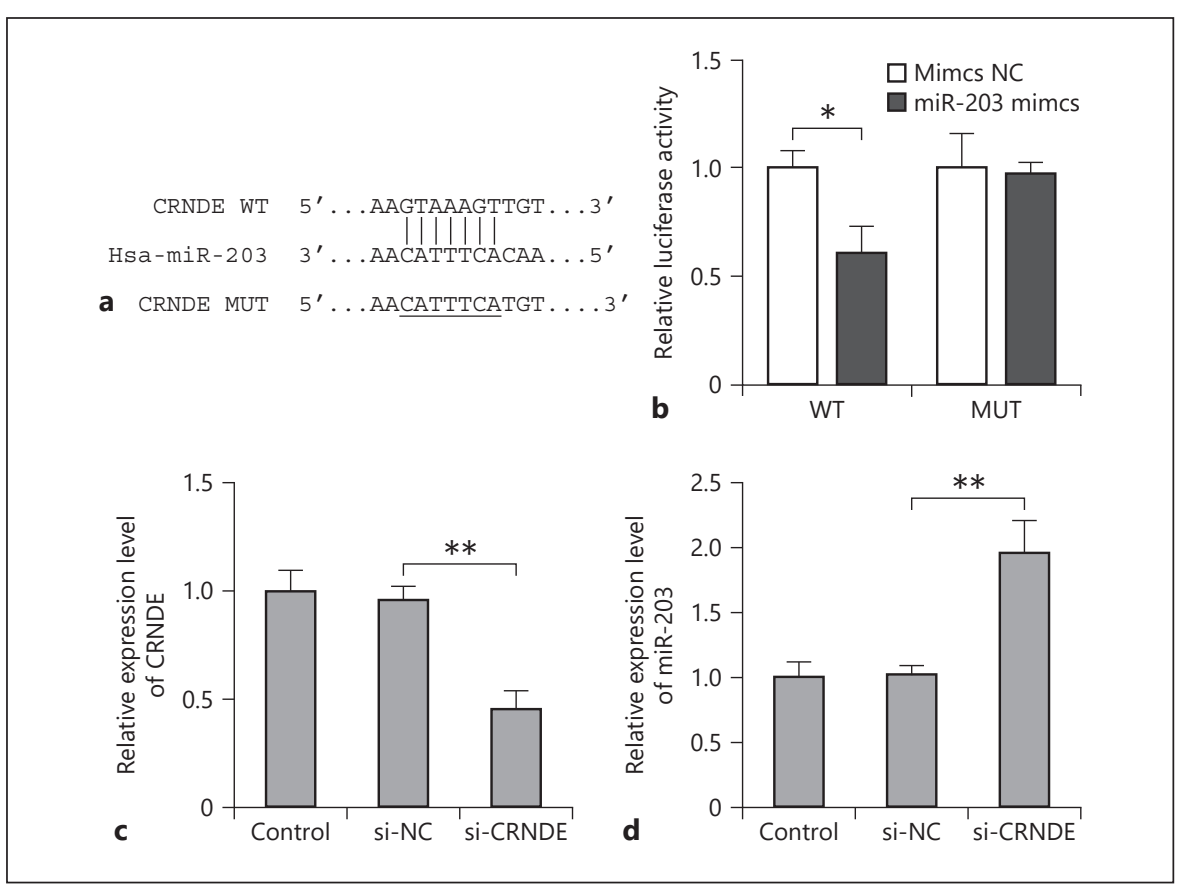

Fig. 5. CRNDE promoted angiogenesis by downregulating miR203/VEGFA axis expression. a qRT-PCR showed that CRNDE was upregulated in $\mathrm{HB}$ tissues compared with para-tumour normal tissues. b Upregulated CRNDE was detected in groups III and IV compared with groups I and II. The results showed that a higher CRNDE level was detected when the tumour size was greater than 5. CRNDE expression was not related to age or gender. c The expression of CRNDE was significantly decreased in HuH-6 cells transfected with si-CRNDE. d Western blotting results showed that VEGFA expression was significantly increased in $\mathrm{HuH}-6$ cells treated with miR-203 inhibitor was decreased in the si-CRNDE group but was partly increased in the si-CRNDE + miR-203 in- hibitor group. e The MTT assay suggested that downregulated CRNDE suppressed the proliferation of HUVECs, but this effect was reversed by co-transfection with si-CRNDE and miR-203 inhibitor. $\mathbf{f}$ The wound healing assay suggested that downregulated CRNDE suppressed the migration of HUVECs, which was reversed by co-transfection with si-CRNDE and miR-203 inhibitor. g The tube formation assay suggested that downregulated CRNDE suppressed the angiogenesis of HUVECs, but the effect was reversed by co-transfection with si-CRNDE and miR-203 inhibitor. h Upregulated CRNDE suppressed the expression of miR-203, and VEGFA expression increased, which led to increased angiogenesis in HUVECs.

(For figure see next page.) 


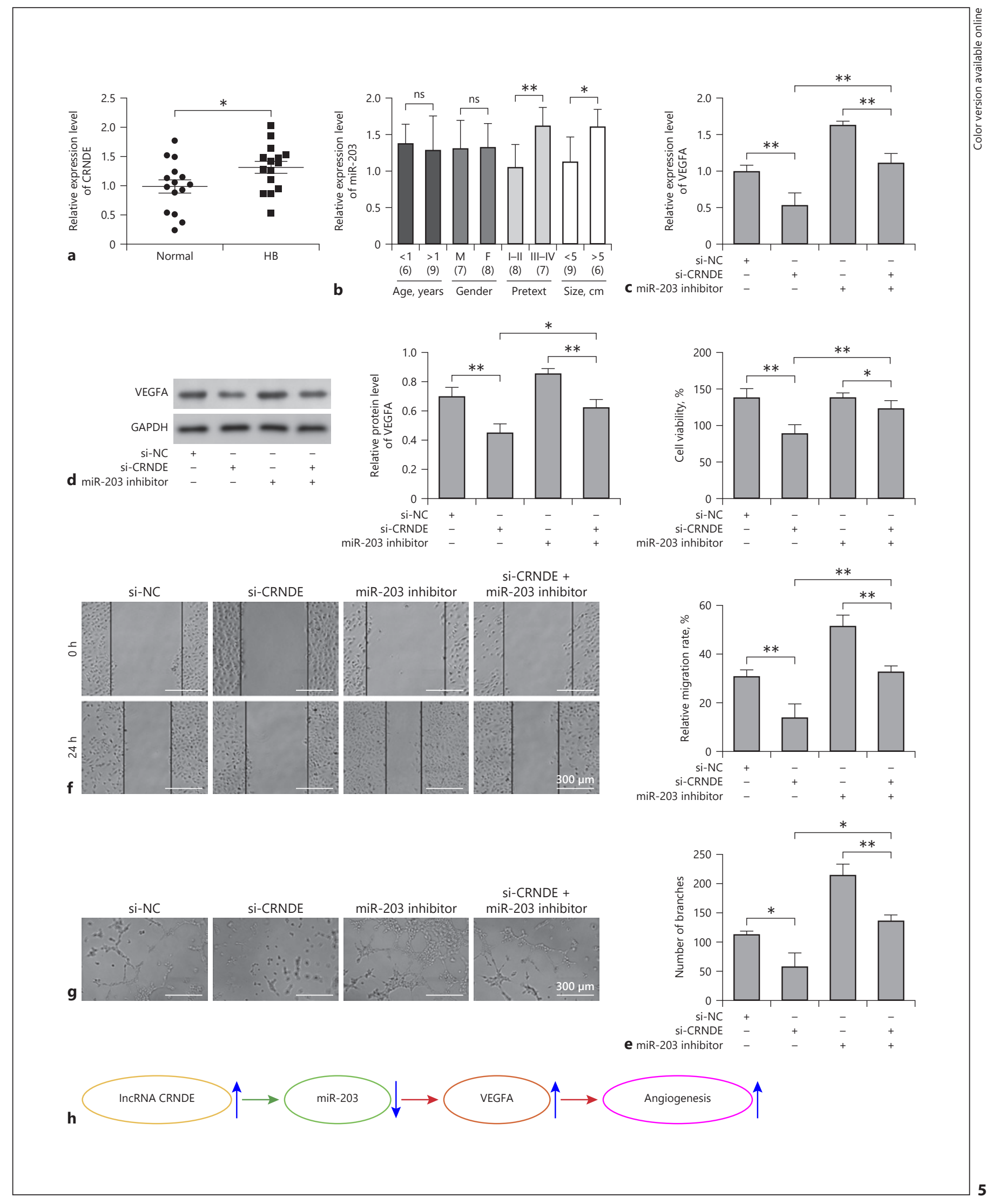


while co-transfection of miR-203 mimics and p-VEGFA could inhibit angiogenesis (Fig. 3d). These results suggested that miR-203 could inhibit angiogenesis by regulating VEGFA expression.

\section{IncRNA CRNDE Binds miR-203 and Negatively}

Regulates miR-203 Expression

Based on the effects of CRNDE on HB development reported by several studies, bioinformatics analysis was performed to analyse the relationship between CRNDE and miR-203. It was found that miR-203 could be a target of CRNDE (Fig. 4a). A luciferase reporter assay was performed to further confirm the relationship between CRNDE and miR-203. As shown in Figure 4b, HuH-6 cells co-transfected with WT-CRNDE and miR-203 mimics showed weaker luciferase activity compared with other groups. qRT-PCR results suggested that the expression of CRNDE was reduced in cells treated with si-CRNDE (Fig. 4c). As shown in Figure 4d, miR-203 expression was significantly increased after transfection of $\mathrm{HuH}-6$ cells with si-CRNDE. The results indicated that CRNDE could target and negatively regulate miR-203 in HuH-6 cells.

\section{IncRNA CRNDE Promotes Angiogenesis via}

Downregulating miR-203 Expression

To explore the roles of CRNDE in HB tissues, qRTPCR was used to measure CRNDE expression in HB cancer tissues and normal adjacent tissues. By qRT-PCR assay, we found that the expression of CRNDE was significantly upregulated in $\mathrm{HB}$ cancer tissues (Fig. 5a). As shown in Figure 5b, CRNDE was upregulated in groups III and IV compared to groups I and II. The results showed that a higher CRNDE level was detected when the tumour size was over $5 \mathrm{~cm}$. CRNDE expression was not related to age or gender. $\mathrm{HuH}-6$ cells were transfected with si-NC, si-CRNDE, and/or miR-203 inhibitor. Using qRT-PCR and Western blotting assays, we found that the expression of VEGFA decreased in the si-CRNDE group, while co-transfection with si-CRNDE and miR-203 inhibitor restored the expression of VEGFA (Fig. 5c, d). Then, the cell supernatant of the HuH-6 cells was collected and added to HUVECs for $24 \mathrm{~h}$. The MTT assay showed that downregulated CRNDE expression significantly inhibited the viability of HUVECs, while upregulated CRNDE expression promoted HUVEC viability, which was blocked by inhibition of miR-203 (Fig. 5e). A wound healing assay indicated that knockdown of CRNDE could suppress the migration of HUVECs, which was reversed by knockdown of miR-203 (Fig. 5f). The tube formation assay suggested that downregulation of
CRNDE significantly inhibited HUVEC angiogenesis, which was reversed by co-transfection of si-CRNDE and miR-203 inhibitor (Fig. 5g). The above results suggested that lncRNA CRNDE could regulate angiogenesis by regulating the expression of miR-203. A summary of these results is shown in a graphical representation (Fig. 5h). Upregulation of CRNDE suppressed the expression of miR-203 and thus increased VEGFA expression, eventually leading to angiogenesis in HUVECs.

\section{Discussion}

$\mathrm{HB}$, the most common primary liver malignancy in children, is usually diagnosed in the first 3 years after birth [2]. Inhibition of tumour growth and angiogenesis is the mainstream mechanism for antitumour drug development [23]. Angiogenesis is an important process for a variety of tumour biological events, including blood supply delivery, tumour growth, invasion, and metastasis, and it also plays an essential role in the tumourigenesis and metastasis of HB [24].

MiR-203 has been proven to be downregulated in multiple malignant tumours, including bladder cancer, prostate cancer, breast cancer, and cervical cancer, as well as hepatocellular carcinoma [17-21]. It was reported that miR-203 could act as a tumour suppressor by inhibiting cell proliferation and angiogenesis in cervical cancer [22]. It has been proven that the expression of miR-203 can be regulated by several molecules. Zheng et al. [25] found that lncRNA AB209371 accelerated the development and progression of ovarian carcinoma by suppressing miR203 expression. MALAT1 promoted the development of renal cell carcinoma by reducing the expression of miR203 expression [26]. Furthermore, miR-203 has been proven to be involved in the expression of several cancerrelated factors. MiR-203 suppressed ovarian carcinoma progression by inhibiting Survivin expression [25] and inhibited epithelial-mesenchymal transition by decreasing Snail and Slug expression, which are regarded as biomarkers of the epithelial-mesenchymal transition process [27]. This evidence suggests that miR-203 could potentially become a cancer therapeutic target and prognostic biomarker. However, whether miR-203 could regulate angiogenesis in $\mathrm{HB}$ remains unclear.

The downregulation of miR-203 has been reported to be associated with several tumours [22, 28]. In our study, we first determined that miR-203 was downregulated in $\mathrm{HB}$ tissues, and miR-203 expression was closely related to tumour size and tumour stage. Higher miR-203 levels 
were detected in tumours with sizes greater than $5 \mathrm{~cm}$. Additionally, downregulated miR-203 was more frequently detected in groups III and IV compared with groups I and II. Because several reports suggested that low expression of miR-203 promoted angiogenesis [22, 29], we hypothesized that miR-203 may be associated with HB pathogenesis and progression. To explore the potential role of $\mathrm{miR}$ 203 in HB, MTT, wound healing, and tube formation assays were performed to determine cell viability, in vitro migration capacity, and angiogenesis. Our data proved that miR-203 inhibited cell angiogenesis.

Vascular endothelial growth factor A (VEGFA), encoded by the VEGFA gene, is a member of the VEGF family. VEGFA has been shown to stimulate endothelial cell migration and angiogenesis, which often occur in $\mathrm{HB}[23$, 24]. At present, multiple studies have confirmed that miRNAs can inhibit tumour progression by regulating the expression of VEGFA. miRNA-205-5p inhibited renal carcinoma cell development by negatively regulating VEGFA expression [30]. Furthermore, downregulated miR-126-3p was proven to be associated with dabrafenib resistance in melanoma by upregulating VEGFA expression [31]. Consistent with a previous study [22, 28, 32], we demonstrated that the $3^{\prime} \mathrm{UTR}$ of the VEGFA transcript might be a direct target of miR-203 in HuH-6 cells. The results of qRT-PCR and Western blotting showed that miR-203 negatively regulated VEGFA expression. Further study revealed that miR-203 suppressed the viability, migration, and angiogenesis of HUVECs by regulating VEGFA expression in $\mathrm{HuH}-6$ cells.

lncRNAs, an important family of noncoding RNAs, are involved in the regulation of gene expression via several mechanisms, especially by acting as endogenous miRNA sponges to affect the biological functions of multiple tumour cell types [33]. Recently, it was found that CRNDE increased HCC cell proliferation and metastasis by suppressing miR-384 expression [34]. In addition, CRNDE contributes to multiple myeloma tumour growth by suppressing miR-451 [35]. Similarly, this study indicated that CRNDE targeted and negatively regulated miR-203 in HuH- 6 cells. It has been reported that lncRNAs play crucial roles in transcription, cell differentiation, tumourigenesis, and metastasis in several human cancers [35]. It was demonstrated that CRNDE was elevated in colorectal cancer and that knockdown of CRNDE could inhibit cell growth and metastasis [36], while upregulation of CRNDE could promote tumour growth and migration in glioma [37]. In line with a previous study, we determined that CRNDE was elevated in $\mathrm{HB}$ tissues and $\mathrm{HuH}-6$ cells. Furthermore, upregulated CRNDE was detected in groups III and IV compared with groups I and I, which indicates that the CRNDE level was related to the tumour stage. Downregulation of CRNDE could significantly inhibit angiogenesis. Furthermore, CRNDE promoted angiogenesis by downregulating miR-203 expression.

In conclusion, IncRNA CRNDE promoted angiogenesis by regulating the expression of the miR-203/VEGFA axis. These findings demonstrated the regulatory mechanism of the CRNDE/miR-203/VEGFA network in HB and laid a foundation for considering miR-203 and CRNDE as novel tumour biomarkers and therapeutic targets for the diagnosis and treatment of $\mathrm{HB}$.

\section{Statement of Ethics}

This study was approved by the Ethics Committee of Hunan Children's Hospital.

\section{Disclosure Statement}

The authors declare that there are no conflicts of interest.

\section{Funding Sources}

The authors have no funding sources to disclose.

\section{Author Contributions}

Qiang Yin and Li-Jian Chen: conception and design of the work. Miao-Xian Yuan, Chun-Yi Ji, and Yu-Ming Peng: interpretation of data and conducting of experiments. Yan-Bing Zhang and Tian Zhang: data acquisition and analysis. Hong-Qiang Gao: drafting the work and conducting of experiments. Zhao-Yang Liu: drafting the work and interpretation of data. Xin-Yi Sheng and Wei-Xin Xie: revising the work critically for important intellectual content. All authors gave final approval of the version to be published.

\section{References}

1 Czauderna P, Haeberle B, Hiyama E, Rangaswami A, Krailo M, Maibach R, et al. The Children's Hepatic tumors International Collaboration (CHIC): novel global rare tumor database yields new prognostic factors in hepatoblastoma and becomes a research model. Eur J Cancer. 2016 Jan;52:92-101.

2 Sharma D, Subbarao G, Saxena R. Hepatoblastoma. Semin Diagn Pathol. 2017 Mar; 34(2): 192-200.

3 Lin Y, Yu J, Wu J, Wang S, Zhang T. Abnormal level of CUL4B-mediated histone H2A ubiquitination causes disruptive HOX gene expression. Epigenetics Chromatin. 2019 Apr;12(1):22. 
4 Wang X, Guo S, Zhao R, Liu Y, Yang G. STAT3-activated long non-coding RNA lung cancer associated transcript 1 drives cell proliferation, migration, and invasion in hepatoblastoma through regulation of the miR301b/STAT3 axis. Hum Gene Ther. 2018; 30(6):702-13.

5 Ecevit CO, Aktaş S, Tosun Yildirim $\mathrm{H}$, Demirağ B, Erbay A, Karaca İ, et al.; MicroRNA-17, MicroRNA-19b, MicroRNA-146a, MicroRNA-302d Expressions in Hepatoblastoma and Clinical Importance. MicroRNA-17, MicroRNA-19b, MicroRNA-146a, MicroRNA-302d Expressions in Hepatoblastoma and Clinical Importance. J Pediatr Hematol Oncol. 2019 Jan;41(1):7-12.

6 Zhao X, Yin H, Li N, Zhu Y, Shen W, Qian S, et al. An integrated regulatory network based on comprehensive analysis of mRNA expression, gene methylation and expression of long non-coding RNAs (lncRNAs) in myelodysplastic syndromes. Front Oncol. 2019 Mar;9: 200.

7 Luan F, Chen W, Chen M, Yan J, Chen H, Yu $\mathrm{H}$, et al. An autophagy-related long non-coding RNA signature for glioma. FEBS Open Bio. 2019 Mar;9(4):653-67.

8 Wang M, Liu Y, Li C, Zhang Y, Zhou X, Lu C. Long noncoding RNA OIP5-AS1 accelerates the ox-LDL mediated vascular endothelial cells apoptosis through targeting GSK-3 $\beta$ via recruiting EZH2. Am J Transl Res. 2019 Mar; 11(3):1827-34

9 Youness RA, Hafez HM, Khallaf E, Assal RA Abdel Motaal A, Gad MZ. The long noncoding RNA sONE represses triple-negative breast cancer aggressiveness through inducing the expression of miR-34a, miR-15a, miR16, and let-7a. J Cell Physiol. 2019 Nov; 234(11):20286-97.

10 Paraskevopoulou MD, Vlachos IS, Karagkouni D, Georgakilas G, Kanellos I, Vergoulis T, et al. DIANA-LncBase v2: indexing microRNA targets on non-coding transcripts. Nucleic Acids Res. 2016 Jan;44 D1:D231-8.

11 Yan H, Liang H, Liu L, Chen D, Zhang Q. Long noncoding RNA NEAT1 sponges miR-125a-5p to suppress cardiomyocyte apoptosis via BCL2L12. Mol Med Rep. 2019 May;19(5):4468-74.

12 Wang X, Guo S, Zhao R, Liu Y, Yang G. STAT3-Activated Long Non-Coding RNA Lung Cancer Associated Transcript 1 Drives Cell Proliferation, Migration, and Invasion in Hepatoblastoma Through Regulation of the miR-301b/STAT3 Axis. Hum Gene Ther. 2019 Jun;30(6):702-13.

13 Dong R, Jia D, Xue P, Cui X, Li K, Zheng S, et al. Genome-wide analysis of long noncoding RNA (lncRNA) expression in hepatoblastoma tissues. PLoS One. 2014 Jan;9(1):e85599.
14 Guo J, Huang X, Wang H, Yang H. Celastrol Induces Autophagy by Targeting $\mathrm{AR} / \mathrm{miR}$ 101 in Prostate Cancer Cells. PLoS One. 2015 Oct;10(10):e0140745.

15 Kristensen H, Haldrup C, Strand S, Mundbjerg K, Mortensen MM, Thorsen K, et al. Hypermethylation of the GABRE miR$452 \sim$ miR-224 promoter in prostate cancer predicts biochemical recurrence after radical prostatectomy. Clin Cancer Res. 2014 Apr; 20(8):2169-81.

16 Lynch SM, O’Neill KM, McKenna MM, Walsh CP, McKenna DJ. Regulation of miR$200 \mathrm{c}$ and miR-141 by Methylation in Prostate Cancer. Prostate. 2016 Sep;76(13):1146-59.

17 Bo J, Yang G, Huo K, Jiang H, Zhang L, Liu D, et al. microRNA-203 suppresses bladder cancer development by repressing bcl-w expression. FEBS J. 2011 Mar;278(5):786-92.

18 Wilting SM, Verlaat W, Jaspers A, Makazaji NA, Agami R, Meijer CJ, et al. Methylationmediated transcriptional repression of microRNAs during cervical carcinogenesis. Epigenetics. 2013 Feb;8(2):220-8.

19 Wang C, Zheng X, Shen C, Shi Y. MicroRNA-203 suppresses cell proliferation and migration by targeting BIRC 5 and LASP 1 in human triple-negative breast cancer cells. J Exp Clin Cancer Res. 2012 Jun;31(1):58.

20 Viticchiè G, Lena AM, Latina A, Formosa A, Gregersen LH, Lund AH, et al. MiR-203 controls proliferation, migration and invasive potential of prostate cancer cell lines. Cell Cycle. 2011 Apr;10(7):1121-31.

21 Furuta M, Kozaki KI, Tanaka S, Arii S, Imoto I, Inazawa J. miR-124 and miR-203 are epigenetically silenced tumor-suppressive microRNAs in hepatocellular carcinoma. Carcinogenesis. 2010 May;31(5):766-76.

22 Zhu X, Er K, Mao C, Yan Q, Xu H, Zhang Y, et al. miR-203 suppresses tumor growth and angiogenesis by targeting VEGFA in cervical cancer. Cell Physiol Biochem. 2013;32(1):64-73.

23 Dong R, Liu XQ, Zhang BB, Liu BH, Zheng S, Dong KR. Long non-coding RNA-CRNDE: a novel regulator of tumor growth and angiogenesis in hepatoblastoma. Oncotarget. 2017 Jun;8(26):42087-97.

24 Dong R, Liu GB, Liu BH, Chen G, Li K, Zheng $\mathrm{S}$, et al. Targeting long non-coding RNATUG1 inhibits tumor growth and angiogenesis in hepatoblastoma. Cell Death Dis. 2016 Jun;7(6):e2278.

25 Zheng ZH, Wu DM, Fan SH, Wen X, Han XR, Wang S, et al. LncRNA AB209371 up-regulated Survivin gene by down-regulating miR203 in ovarian carcinoma. J Ovarian Res. 2019 Oct;12(1):92.

26 Zhang H, Li W, Gu W, Yan Y, Yao X, Zheng J. MALAT1 accelerates the development and progression of renal cell carcinoma by decreasing the expression of miR-203 and promoting the expression of BIRC5. Cell Prolif. 2019 Sep;52(5):e12640.
27 Jiang L, Wang R, Fang L, Ge X, Chen L, Zhou $\mathrm{M}$, et al. HCP5 is a SMAD3-responsive long non-coding RNA that promotes lung adenocarcinoma metastasis via miR-203/SNAI axis. Theranostics. 2019 Apr;9(9):2460-74.

$28 \mathrm{Xu} \mathrm{L}$, Shen B, Chen T, Dong P. miR-203 is involved in the laryngeal carcinoma pathogenesis via targeting VEGFA and Cox-2. OncoTargets Ther. 2016 Jul;9:4629-37.

29 Tian X, Tao F, Zhang B, Dong JT, Zhang Z. The miR-203/SNAI2 axis regulates prostate tumor growth, migration, angiogenesis and stemness potentially by modulating GSK- $3 \beta /$ $\beta$-CATENIN signal pathway. IUBMB Life. 2018 Mar;70(3):224-36.

30 Huang J, Wang X, Wen G, Ren Y. miRNA205-5p functions as a tumor suppressor by negatively regulating VEGFA and PI3K/Akt/ mTOR signaling in renal carcinoma cells. Oncol Rep. 2019 Nov;42(5):1677-88.

31 Caporali S, Amaro A, Levati L, Alvino E, Lacal PM, Mastroeni S, et al. miR-126-3p downregulation contributes to dabrafenib acquired resistance in melanoma by up-regulating ADAM9 and VEGF-A. J Exp Clin Cancer Res. 2019 Jun;38(1):272.

32 Liu F, Wu W, Wu K, Chen Y, Wu H, Wang H, et al. MiR-203 Participates in Human Placental Angiogenesis by Inhibiting VEGFA and VEGFR2 Expression. Reprod Sci. 2018 Mar; 25(3):358-65.

33 Hirata H, Hinoda Y, Shahryari V, Deng G, Nakajima K, Tabatabai ZL, et al. Long Noncoding RNA MALAT1 Promotes Aggressive Renal Cell Carcinoma through Ezh2 and Interacts with miR-205. Cancer Res. 2015 Apr; 75(7):1322-31.

34 Chen Z, Yu C, Zhan L, Pan Y, Chen L, Sun C. LncRNA CRNDE promotes hepatic carcinoma cell proliferation, migration and invasion by suppressing miR-384. Am J Cancer Res. 2016 Oct;6(10):2299-309.

35 Meng YB, He X, Huang YF, Wu QN, Zhou YC, Hao DJ. Long Noncoding RNA CRNDE Promotes Multiple Myeloma Cell Growth by Suppressing miR-451. Oncol Res. 2017 Aug; 25(7):1207-14

36 Yu B, Ye X, Du Q, Zhu B, Zhai Q, Li XX. The Long Non-Coding RNA CRNDE Promotes Colorectal Carcinoma Progression by Competitively Binding miR-217 with TCF7L2 and Enhancing the Wnt/ $\beta$-Catenin Signaling Pathway. Cell Physiol Biochem. 2017;41(6): 2489-502.

37 Wang Y, Wang Y, Li J, Zhang Y, Yin H, Han B. CRNDE, a long-noncoding RNA, promotes glioma cell growth and invasion through mTOR signaling. Cancer Lett. 2015 Oct;367(2):122-8. 